\title{
Pharmacy Participation in Non-Prescription Syringe Sales in Los Angeles and San Francisco Counties, 2007
}

Erin N. Cooper, Chaka Dodson, Thomas J. Stopka, Elise D. Riley, Richard S. Garfein, and Ricky N. Bluthenthal

\begin{abstract}
Increasing sterile syringe access for injection drug users (IDUs) is one way to prevent HIV and hepatitis C virus (HCV) transmission in this population. In 2005, California Senate Bill 1159 allowed counties to adopt the Disease Prevention Demonstration Project (DPDP). Where enacted, the DPDP allows pharmacies that register with the county to sell up to ten syringes to adults without a prescription. In the current study, we describe pharmacy participation in nonprescription syringe sales (NPSS) in two counties in California and examine factors associated with NPSS. Telephone and in-person interviews were conducted in Los Angeles (LA) and San Francisco (SF) with 238 pharmacies in 2007 ( $n=67$ in SF; $n=171$ in LA). Quantitative survey items captured pharmacy registration with the county, pharmacy policies/ practices, episodes and conditions of NPSS and refusals to sell, potential negative consequences of NPSS, and staff attitudes regarding HIV and HCV prevention for IDUs. Overall, $42 \%$ of pharmacies reported NPSS $(28 \%$ in LA and $81 \%$ in SF), although only $34 \%$ had registered with the county (17\% in LA and $76 \%$ in SF). Many pharmacies required proof of a medical condition $(80 \%$ in LA and $30 \%$ in SF) and refused NPSS if the customer was a suspected IDU (74\% in LA, 33\% in SF). Few negative consequences of NPSS were reported. In multivariate logistic regression analysis, we found that the odds of NPSS were significantly higher among pharmacists who thought syringe access was important for preventing HIV among IDUs [adjusted odds ratio $(A O R)=2.95 ; 95 \%$ confidence interval $(C I)=1.10-7.92]$, were chain pharmacies $(A O R=12.5 ; 95 \% C I=4.55-33.33)$, and were located in $S F(A O R=4.88$; $95 \% C I=1.94-12.28)$. These results suggest that NPSS were influenced by pharmacists' perception. NPSS might be increased through greater educational efforts directed at pharmacists, particularly those in non-chain pharmacies.
\end{abstract}

KEYWORDS HIV, Viral hepatitis, Sterile syringe access, Pharmacy, Policy, Evaluation

Cooper, Dodson, and Bluthenthal are with the Urban Community Research Center, Sociology Department, California State University Dominguez Hills, Carson, CA, USA; Cooper and Dodson are with the Psychology Department, California State University Dominguez Hills, Carson, CA, USA; Stopka is with the California Department of Public Health, Office on AIDS, Sacramento, CA, USA; Riley is with the Department of Medicine, University of California, San Francisco, CA, USA; Garfein is with the Department of Medicine, University of California San Diego School of Medicine, San Diego, CA, USA; Bluthenthal is with the Health Program and Drug Policy Research Center, RAND Corporation, Sta. Monica, CA, USA.

Correspondence: Ricky N. Bluthenthal, PhD, Urban Community Research Center, Sociology Department, California State University Dominguez Hills, 1000 East Victoria Street, Carson, CA 90747, USA. (E-mail: rickyb@rand.org) 


\section{INTRODUCTION}

Illicit drug injection accounts for a significant proportion of HIV/AIDS cases and the majority of hepatitis $\mathrm{C}$ virus (HCV) cases in the USA. ${ }^{1,2}$ To prevent the spread of HIV and HCV, federal officials have recommended that injection drug users (IDUs) inject with a clean, unused syringe each time they use. ${ }^{3-5}$ To achieve this recommendation, IDUs must have access to clean syringes. In the USA, clean syringes can be obtained through nonprescription syringe sales (NPSS) at pharmacies or through syringe exchange programs (SEPs).

A variety of studies indicate that pharmacy access to sterile syringes could have a significant impact on efforts to prevent HIV and HCV among IDUs. ${ }^{6,7}$ While the entire USA had $<200$ SEPs in $2005,{ }^{8}$ California had over 7,000 licensed pharmacies in 2007, but only 17 SEP sites in San Francisco and 24 in Los Angeles. In addition, as compared to SEPs, pharmacies are neither politically controversial nor do they require funding from government and/or foundations to operate. ${ }^{9,10}$ Lastly, pharmacy access to syringes has been shown to be effective at lowering injectionrelated HIV risk behaviors among the IDUs who use them. ${ }^{11-13}$

However, not all pharmacies are willing to conduct NPSS. Rural pharmacists in Connecticut, Missouri, Kentucky, and Colorado were less supportive of NPSS than their urban counterparts. ${ }^{14}$ Chain pharmacies have been found to be more likely to sell syringes than independent pharmacies. ${ }^{12,15,16}$ Furthermore, pharmacy managers and supervisors as well as pharmacists with ten or more years of experience have been found to be more likely to support NPSS. ${ }^{15}$ Lastly, pharmacists who viewed drug use and addiction as public health issues were more likely to support NPSS than those who regarded addiction as a personal choice. ${ }^{17,18}$ These studies underscore that permitting NPSS does not guarantee pharmacy participation. However, pharmacy participation in NPSS is critical if this method of sterile syringe access is to have its intended impact on preventing HIV and HCV infection among IDUs.

An additional impediment to pharmacy syringe sales is medical prescription laws that prohibit pharmacies from selling syringes to adults without a doctor's prescription. California is one of only a few states that have a medical prescription law for syringes. In 2005, the state legislature and governor enacted Senate Bill (SB) 1159 to amend this law through the establishment of Disease Prevention Demonstration Projects (DPDP). For county and city governments that opt-in to the DPDP, pharmacies that register with the local government can sell up to ten syringes to adults (18 years of age or older) without a medical prescription. In addition, local governments can require pharmacies that participate in the DPDP to provide information on syringe disposal, drug treatment, infectious disease testing, and other medical and social services. Lastly, in DPDP locales, adults may legally possess up to ten syringes without a prescription provided that these syringes were obtained from an authorized source (i.e., registered pharmacy or legal SEP). More details on the DPDP are available elsewhere. ${ }^{19}$

As we have noted, variability in pharmacy participation should be expected. In this paper, we examine DPDP implementation in two large and important counties in California-Los Angeles (LA) and San Francisco (SF). While limited funding and logistical concerns influenced the selection of these two counties, it is also worth noting that SF can be classified as an early adopter of syringe access and other health interventions for IDUs. In SF, SEPs were implemented in 1989, the DPDP was adopted in 2005, and Naloxone distribution for the reversal of heroin overdoses 
was initiated in 2003. By contrast, SEPs were not implemented and approved in LA until 1995, the DPDP was not adopted until early 2007, and county-approved Naloxone distribution did not start until 2007. In the following, we will present data describing differences in NPSS uptake and practices in LA and SF.

We were also interested in a number of other issues including whether implementation of NPSS was associated with any unintended or negative consequences. And lastly, we conducted analyses to determine what factors (including city, pharmacy characteristics, and pharmacists' attitudes and beliefs) were associated with NPSS in the overall sample of pharmacies in our study.

\section{METHODS}

Data for this study came from quantitative surveys conducted with pharmacies in SF and LA Counties. Licensed pharmacies as of February 1, 2007 were identified from a list obtained from the California Board of Pharmacy. Surveys were restricted to retail pharmacies, thus excluding those that were not open to the general public. All retail pharmacies in SF $(N=132)$ were originally included in the sample. However, during recruitment, we discovered that 27 pharmacies had closed, moved without providing forwarding information, or discontinued the pharmacy component of their business, leaving our overall sample size for SF at 106. Since LA County had over 1,700 retail pharmacies in 2007 and we lacked the resources to contact and interview all of them, we restricted our LA sample in the following ways. First, we selected three special planning areas (SPAs) in LA County that had the highest rates of injection-related HIV/AIDS. ${ }^{20}$ Within these three SPAs $(4,6$, and 8), using data from the LA Alcohol and Drug Program, we calculated the total number of IDUs in treatment and average number of IDUs in treatment for each zip code from 2004 to 2006. Zip codes with both above average counts of IDUs and above average IDUs in treatment per capita were identified $(N=100)$. From this list, 33 zip codes were selected with the highest counts and average. In these zip codes, 211 pharmacies were listed and efforts were made to recruit all eligible pharmacies.

Each selected pharmacy was sent an informational letter and received a follow-up call 1 week later, during which the phone interviewer asked to speak with the pharmacy manager or another person who was knowledgeable about pharmacy practices (e.g., pharmacy technicians). Various methods were used to improve response rates, including in-person interviews in LA and mailed and faxed surveys (in both locations). Using these methods, 67 (or $64 \%$ of total) surveys were completed in SF and 171 (or $81 \%$ of total) were completed in LA, for an overall response rate of $75 \%$.

After completing verbal informed consent, pharmacists were asked a series of questions about their participation in the local DPDP, including registration and syringe sales; conditions of syringe sales; any refusals of sales and the reasons for it; knowledge, attitudes, and beliefs about syringe access; and any perceived problems related to NPSS. For completing the survey, pharmacists received a $\$ 10$ bookstore gift card. All study procedures were reviewed and approved by the Institutional Review Board at California State University Dominguez Hills and the Committee for the Protection of Human Subjects, Health and Human Services Agency, State of California.

\section{Study Measures}

Pharmacies were designated as registered or not registered to sell syringes with their local DPDP as well as whether they had actually sold syringes without a prescription 
(referred to as NPSS hereafter) in the past 12 months. Frequency of providing information on drug treatment, HIV, and HCV testing, syringe disposal, and needle exchange was assessed among pharmacies that reported NPSS, as was the frequency of NPSS during January 2007 and the estimated proportion of NPSS purchasers who were IDUs. The survey also assessed syringe disposal options, requirements for syringe sales, and syringe sale refusals. Pharmacists were also asked if they placed conditions on NPSS beyond verifying the age of customers.

Questions regarding potential unintended or negative consequences of NPSS were asked, including the frequency of problems (many, some, few, or no problems) and whether the following incidents had occurred related to nonprescription pharmacy sales: underage person attempting to buy syringes, verbal or other abuse by persons, increase in the amount of used syringes found on premises, increase in the amount of injection drug use on the premises, increase in shoplifting or other thefts, customer complaints, community opposition, and increase in condom sales.

To identify factors associated with NPSS, we assessed pharmacists' attitudes toward NPSS as well as pharmacy type [chain, independent, care (i.e., Kaiser or community clinic-based facility)], location (free standing, mall or shopping center, in hospital or clinic, in grocery store), and county (LA vs. SF). For attitudes, we used a Likert scale (strongly agree, agree, not sure, disagree, strongly disagree) to assess endorsement of the following items: "Injection drug users will continue to share syringes at the same rate even if they have increased access to clean syringes;" "It is important to provide clean syringes to people who cannot stop injecting drugs;" "Pharmacy access to syringes is an important public health measure;" "An increase in syringe access will increase the number of syringes found on the street and on playgrounds;" "HIV/AIDS is an important health concern in the area served by my pharmacy;" and "Hepatitis C virus infection is an important concern in the area served by my pharmacy." To facilitate analysis, we recoded these response items to agree which includes strongly agree and agree vs. all other responses (not sure, disagree, strongly disagree).

\section{Statistical Analysis}

Frequencies and proportions were used to describe the sample population and central tendencies. Chi-square and Fisher's exact test indicated significant bivariate associations between pharmacy participation and practices related to NPSS. To determine factors independently associated with reporting at least one NPSS in the last 12 months, we conducted multivariate logistic regression with pharmacists' attitudes and characteristics (i.e., years at the pharmacy), pharmacy characteristics (including type-chain, independent, or care), and county as potential predictors. In this model, we included factors found to be significant in bivariate analysis at the $p<$ 0.05 level. We also reran multivariate logistic regression for NPSS participation and enrollment in DPDP separately for each site.

\section{RESULTS}

Two-hundred thirty-eight surveys were completed (67 in SF and 171 in LA). Overall, $52 \%$ of pharmacies were chain stores, $43 \%$ were independents, and $5 \%$ were care pharmacies. In terms of location, $45 \%$ were free-standing retail pharmacies, $20 \%$ were inside a grocery store, $20 \%$ were inside a mall, and $15 \%$ were located in a hospital or clinic. 
In our sample, only $34 \%(17 \%$ in LA and $76 \%$ in SF) of the pharmacies reported being registered with the county as required by the DPDP. However, a total of $42 \%$ of pharmacies reported NPSS in the last 12 months $(28 \%$ in LA and $81 \%$ in $\mathrm{SF})$, suggesting that some pharmacies sold without being registered. Based on estimates provided by respondents, in January 2007, a total of 13,715 syringes (range 10-7,100) were sold in SF and 4,490 (range 10-1,000) were sold in LA. The estimated proportion of IDUs among syringe buying customers was $66 \%$ in SF and $16 \%$ in LA $(p<0.001)$. Due to the consistent differences by county in pharmacy participation, we present the remaining data on pharmacy participation by county.

Information provided (i.e., pamphlets) and syringe disposal options offered by pharmacists that conducted NPSS in the last 12 months differed by county (Table 1). A higher proportion of pharmacies in SF offered information about syringe disposal ( $54 \%$ in LA and $91 \%$ in SF) and SEPs $(28 \%$ in LA and $76 \%$ in SF) compared to those in LA. In terms of syringe disposal, a higher proportion of SF pharmacies provided free sharps containers (11\% in LA and $76 \%$ in SF), on-site syringe drop box (9\% in LA and $46 \%$ in SF), and acceptance of personal sharps containers $(13 \%$ in LA and $73 \%$ in SF). A higher proportion of LA pharmacies offered sharps containers for purchase $(76 \%$ in LA and $38 \%$ in SF). No county differences were observed in the availability of mail-back sharps containers, providing counseling on syringe disposal, and referring customers to other syringe disposal locales.

Table 2 presents data on the type of requirements pharmacies used before conducting a NPSS. The most prevalent requirement was proof of medical condition ( $80 \%$ in LA and $30 \%$ in SF), followed by logging customer name (70\% in LA and $22 \%$ in SF). In all cases, requirements were used more frequently in LA.

We also assessed whether NPSS requests were refused and the reasons for the refusal. Overall, 39\% reported never refusing, 36\% reported rarely refusing, and $25 \%$ reported sometimes or often refusing. County differences were noted, however, with $87 \%$ of SF pharmacies reporting never or rarely refusing to sell syringes, while

TABLE 1 Information and syringe disposal options provided by pharmacies that sold syringes without a prescription during the past 12 months in Los Angeles and San Francisco, January $2007(n=101)$

\begin{tabular}{lcc}
\hline & Los Angeles $(n=47)$ & San Francisco $(n=54)$ \\
\hline Information given & 39 & 56 \\
Drug treatment (\%) & 34 & 47 \\
HIV/HCV prevention and testing (\%) & 28 & 76 \\
Syringe exchange program* (\%) & 54 & 91 \\
Syringe disposal* (\%) & & \\
Syringe disposal options & 11 & 76 \\
Free sharps containers* (\%) & 9 & 46 \\
Drop box* (\%) & 76 & 38 \\
Sharps containers for sale* (\%) & 20 & 24 \\
Mail-back containers (\%) & 13 & 73 \\
Accepts sharps containers* (\%) & 11 & 15 \\
Accepts loose syringes (\%) & 22 & 64 \\
Literature on syringe disposal options* (\%) & 56 & 67 \\
Counseling on syringe disposal (\%) & 47 & 33 \\
Referral to other locations for disposal (\%) & & \\
\hline
\end{tabular}


TABLE 2 Conditions for nonprescription syringe sales in LA and SF, $2007(n=101)$.

\begin{tabular}{lcc}
\hline & Los Angeles $(n=47)$ & San Francisco $(n=54)$ \\
\hline Requirements & & 30 \\
Medical condition** (\%) & 80 & 19 \\
Picture identification** (\%) & 54 & 17 \\
Customer name** (\%) & 63 & 13 \\
Justification* (\%) & 39 & 22 \\
Log customers** (\%) & 70 & \\
Reasons for refusals & & 11 \\
Unfamiliar customer** (\%) & 65 & 11 \\
Known IDU** (\%) & 72 & 4 \\
Disheveled appearance** (\%) & 35 & 32 \\
Intoxication** (\%) & 78 & 26 \\
Excessive purchases* (\%) & 46 & 43 \\
Safety concerns* (\%) & 67 & 19 \\
Concerns about unsafe disposal* (\%) & 46 & 26 \\
Risk of theft* (\%) & 46 & \\
\hline
\end{tabular}

${ }^{*} p<0.05 ;{ }^{* *} p<0.001$

in LA, $60 \%$ reported never or rarely refusing to sell. In LA, another $33 \%$ also reported sometimes refusing to sell syringes.

Table 2 also reports reasons cited by pharmacies for not selling syringes. Differences by county were found for all reasons, with LA pharmacies more likely to endorse every reason for refusing than their SF counterparts. The most common reasons for refusing NPSS in LA was customer intoxication followed by known IDU and safety concerns, while in SF, the most frequently endorsed item was safety concerns, followed by intoxication. SF pharmacists also endorsed excessive purchases and risk of theft as reasons for not selling syringes.

We also explored how pharmacist's knowledge, attitudes and beliefs, pharmacy type, and pharmacy location might influence NPSS decisions. Pharmacists who thought NPSS would be effective, that syringe access was important for HIV prevention, and that HIV is a problem in their area were significantly more likely to have sold syringes (Table 3). Chain pharmacies and those located in grocery stores were more likely to report NPSS as compared to pharmacies in care settings. In multivariate analysis, we found that endorsement of syringe access was important for HIV prevention [adjusted odds ratio $(\mathrm{AOR})=2.95,95 \%$ confidence interval $(\mathrm{CI})=1.10-7.92]$, chain pharmacy $(\mathrm{AOR}=12.5 ; 95 \% \mathrm{CI}=4.55-33.33)$, and being in $\mathrm{SF}(\mathrm{AOR}=4.88 ; 95 \% \mathrm{CI}=1.94-12.28)$ were independently associated with providing NPSS. DPDP registration was not significantly associated with NPSS in the last 12 months.

Due to the consistent differences by county, we constructed models of NPSS for each site. In Los Angeles, we found both endorsement of syringe access as being important for HIV prevention $(p=0.042)$ and chain pharmacy $(p<0.001$ as compared to independent pharmacy) as statistically significant predictors of NPSS. In SF, only chain pharmacy $(p<0.001)$ predicted NPSS.

Lastly, we looked at reported problems related to NPSS. Roughly $60 \%$ of pharmacists reported no problems at all. In addition, the problem cited most $(\sim 25 \%)$ in both LA and SF was verbal abuse by buyers. Approximately $15 \%$ of pharmacists also reported theft in the store as a problem. Levels of criminal activity 
TABLE 3 Bivariate analysis of factors significantly associated with one or more nonprescription syringe sales during the past year in LA and SF, $2007(n=238)$

\begin{tabular}{|c|c|c|}
\hline & No sales $(n=137)$ & Sales $(n=101)$ \\
\hline \multicolumn{3}{|l|}{ Pharmacists' attitudes and belief } \\
\hline 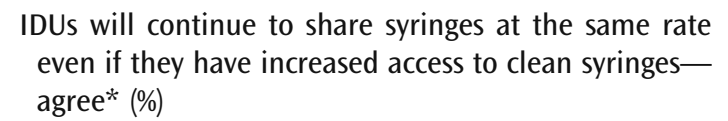 & 43 & 30 \\
\hline $\begin{array}{l}\text { It is important to provide clean syringes to people who } \\
\text { cannot stop injecting drugs-agree* }(\%)\end{array}$ & 68 & 86 \\
\hline $\begin{array}{l}\text { Pharmacy access to syringes is an important public } \\
\text { health measure-agree* }(\%)\end{array}$ & 83 & 93 \\
\hline $\begin{array}{l}\text { An increase in syringe access will increase the number } \\
\text { of syringes found on the street and on playgrounds- } \\
\text { agree* }(\%)\end{array}$ & 55 & 37 \\
\hline $\begin{array}{l}\text { HIV/AIDS is an important health concern in the area } \\
\text { served by my pharmacy-agree* }(\%)\end{array}$ & 72 & 83 \\
\hline \multicolumn{3}{|l|}{ Pharmacy type } \\
\hline Chain $(n=120)^{* *}(\%)$ & 32 & 68 \\
\hline Independent $(n=99)(\%)$ & 87 & 13 \\
\hline Care $(n=10)(\%)$ & 90 & 10 \\
\hline \multicolumn{3}{|l|}{ Pharmacy setting } \\
\hline In grocery-Yes* (\%) & 40 & 60 \\
\hline No (\%) & 62 & 38 \\
\hline In hospital or clinic-Yes ${ }^{* *}(\%)$ & 85 & 15 \\
\hline No (\%) & 53 & 47 \\
\hline
\end{tabular}

${ }^{*} p<0.05 ;{ }^{* *} p<0.001$

and illegal drug activity in the areas with and without NPSS did not differ significantly based on pharmacies reports.

\section{DISCUSSION}

Two years after the implementation of SB1159, substantial differences were observed in the implementation of NPSS in LA and SF. SF pharmacies were more likely to conduct NPSS and less likely to impose requirements on NPSS than their LA counterparts. These differences may be explained by a number of factors including the earlier start of DPDP in SF (2005 as compared to 2007 in LA), higher per capita HIV rates in SF as compared to LA, and/or differences in political attitudes and priorities. This study, and others like it, indicates that detailed research on adoption of NPSS among pharmacies is needed to determine how to improve pharmacy participation in these important public health strategies.

We also found that within NPSS, a number of operational barriers are apparent. In LA, the current level of implementation $(<30 \%$ of pharmacies participate) and the reports of NPSS refusals suggest that syringe access through pharmacies could be increased. With time, this may occur, as has occurred in SF. Given that pharmacists' attitudes were related to NPSS participation, one way to increase pharmacy participation might be through educational programs. Other investigators have recommended pharmacist education as one way to increase participation in NPSS. ${ }^{18,21,22}$ In fact, at least one study has found that efforts to inform community members and pharmacists about the importance of sterile syringe access appeared to 
have positive impacts on pharmacy participation in NPSS in New York. ${ }^{12}$ Such efforts should be considered in areas where pharmacy participation rates appear low.

Another important implication of this research regards the potential negative consequences of this policy. As in other studies, ${ }^{23}$ most pharmacists reported no problems related to NPSS, although evaluation of more objective information such as police calls and arrests could further elucidate these issues. As part of our overall efforts to study the potential negative impacts of this policy, we have also collected data from pharmacists in 2008 as well as conducted physical observations of streets around pharmacies. We will present the results of these data in subsequent reports.

These study results should be considered in light of the following limitations. Interviews were completed with a variety of pharmacy personnel (i.e., managers, technicians), some of whom may not have had complete information on relevant pharmacy policies or practices. However, it is worth noting that rates of participation in NPSS in SF are similar to those found in another survey using mailed questionnaires. ${ }^{24}$ In terms of our attitude items, these were asked of individuals and may not reflect the overall attitudes of other professional staff in a pharmacy. As with all self-reported data, there are concerns regarding socially desirable responding and recall bias. For some items, we also asked pharmacists to estimate activities such as syringes sold or frequency of refusing to sell syringes; in those cases, it is impossible to know the accuracy of these estimates.

There are a number of issues related to sampling and response rates that should also be considered. Our sampling frames and recruitment methods differed by county due to resources and geographic proximity. The large number of pharmacies in LA County $(\sim 1,700)$ prevented us from attempting to contact them all, and so the LA sample should not be considered representative of all pharmacies in LA County. Furthermore, in LA, we focused on a subsample of pharmacies in high-need areas. This difference in sampling frame may have influenced our outcomes. Some studies have found that pharmacies in high need areas (i.e., elevated IDU and HIV rates) were more likely to participate in NPSS than those in lower need areas. ${ }^{25}$ So our site comparison may well be overestimating LA pharmacy participation in NPSS.

Lastly, because of geographic proximity, we were able to also conduct in-person recruitment and interviews at pharmacies in LA. This contributed to our differential response rates $(63 \%$ in SF vs. $81 \%$ in LA). We conducted two additional analyses to account for this difference. First, to determine if site differences in NPSS participation were due to response rates differences, we recalculated NPSS participation for SF, assuming it had the same response rates as LA $(81 \%)$ and that all non-responders reported not participating in NPSS (the most conservative assumption). In this analysis, we found that the NPSS participation rate in SF would be $59 \%(54 / 86)$ and that as compared to LA $(28 \%)$, this difference would still be significant $(p<0.0001)$ using Pearson chi-square test. Second, we reran the multivariate logistic regression predicting NPSS participation separately by site. In LA, we found that both endorsement of the importance of syringe access and chain pharmacy predicted NPSS participation, while in SF, only pharmacy type significantly predicted NPSS participation. However, it is worth noting that we did not have any care pharmacies in our SF site and that the point estimate of the importance of syringe access was in the predictive direction in SF as well. The smaller sample size in SF also impacted this reanalysis. We conclude that chain pharmacy involvement has been key in expanding NPSS and that pharmacists' attitudes in LA are an important contributor to NPSS involvement. Why chain 
pharmacy participation rates are higher was not an issue we could address given our methods and resources.

Substantial legal and policy changes to prevent HIV and hepatitis $\mathrm{C}$ virus infection among IDUs have been made in the last decade. However, as laws and policies are changed to promote infectious disease prevention for IDUs, attention must be paid to the implementation of these policy changes, particularly for NPSS where adoption has been found to vary inversely with need in a few cases. ${ }^{22,26}$ Our results indicate that there were substantial differences in the adoption of NPSS in our two study sites. More studies that examine the process and outcome evaluations of policy changes are needed, as are studies of potential negative consequences of these changes. In addition, to maximize the impact of this policy change, additional efforts appear needed to increase local acceptability, thus broadening implementation among and within counties. In LA and SF, NPSS might be increased through greater educational efforts directed at pharmacists, particularly those who work in independent pharmacies.

\section{ACKNOWLEDGMENTS}

This study was funded by the Office on AIDS, California Department of Public Health (grant no. 06-55589). We appreciate the participation of pharmacy staff in Los Angeles and San Francisco as well as the assistance of student volunteers and Urban Community Research Center Staff, including Heather Guentzel, M.A., MPH, Sandra Chaidez, Charles Hohm, Ph.D., Michelle Carter, Joi Dudley, Rigo Lopez, Erin Lowery, Sonia Ortega, DeAndre Pryor, and Juan Villarreal on this project. The views presented in this study are those of the authors and do not necessarily represent the policy or perspectives of the Office on AIDS, California Department of Public Health or the State of California.

OPEN ACCESS This article is distributed under the terms of the Creative Commons Attribution Noncommercial License which permits any noncommercial use, distribution, and reproduction in any medium, provided the original author(s) and source are credited.

\section{REFERENCE}

1. Armstong GL, Wasley A, Simard EP, McQuillan GM, Kuhnert WL, Alter MJ. The prevalence of hepatitis C virus infection in the United States: 1999 through 2002. Ann Intern Med. 2006;144:705-714.

2. CDC. HIV prevelance estimates-United States, 2006. MMWR. 2008;57(39):1073-1076.

3. CDC. Recommendations for prevention and control of hepatitis $\mathrm{C}$ virus (HCV) infection and HCV-related chronic disease. MMWR Recomm Rep. 1998;47:1-39.

4. CDC. New attitudes \& strategies: a comprehensive approach to preventing blood-borne infections among IDUs. Atlanta: Centers for Disease Control and Prevention; 2000.

5. US Public Health Service. HIV Prevention Bulletin: Medical Advice for Persons Who Inject Illicit Drugs. Atlanta, GA: US Department of Health and Human Services; 1997.

6. Lurie P, Jones TS, Foley J. A sterile syringe for every drug user injection: how many injections take place annually, and how might pharmacists contribute to syringe distribution? J Acquir Immune Defic Syndr. 1998;18(Suppl 1):S45-S51.

7. Taussig JA, Weinstein B, Burris S, Jones TS. Syringe law and pharmacy regulations are structural constraints on HIV prevention in the U.S. AIDS. 2000;14(Suppl 1):S47-S51.

8. CDC. Syringe exchange programs-United States, 2005. MMWR Morb Mortal Wkly Rep. 2007;56(44):1164-1167. 
9. Bluthenthal RN, Heinzerling KG, Anderson R, Flynn NM, Kral AH. Approval of syringe exchange programs in California: results from a local approach to HIV prevention. Am J Public Health. 2008;98(2):278-283.

10. Tempalski B, Friedman R, Keem M, Cooper H, Friedman SR. NIMBY localism and national inequitable exclusion alliances: the case of syringe exchange programs in the United States. Geoforum. 2007;38(6):1250-1263.

11. Cotten-Oldenburg NU, Carr P, DeBoer JM, Collison EK, Novotny G. Impact of pharmacy-based syringe access on injection practices among injecting drug users in Minnesota, 1998 to 1999. J Acquir Immune Defic Syndr. 2001;27:183-192.

12. Fuller C, Galea S, Caceres W, Blaney S, Sisco S, Vlahov D. Multilevel community-based intervention to increase access to sterile syringes among injection drug users through pharmacy sales in New York City. Am J Public Health. 2007;97(1):117-124.

13. Pouget ER, Deren S, Fuller CM, et al. Receptive syringe sharing among injection drug users in Harlem and the Bronx during the New York State expanded syringe access demonstration program. J Acquir Immune Defic Syndr. 2005;39(4):471-477.

14. Reich W, Compton WM, Horton JC, et al. Pharmacist ambivalence about sale of syringes to injection drug users. J Am Pharm Assoc (Wash). 2002;42(6 Suppl 2):S52-S57.

15. Diebert R, Goldbaum G, Parker T, et al. Increased access to unrestricted pharmacy sale of syringes in Seattle-King County, Washington: structural and individual level changes. Am J Public Health. 2006;96(8):1347-1353.

16. Farley TA, Niccolai LM, Billeter M, Kissinger PJ, Grace M. Attitudes and practices of pharmacy managers regarding needle sales to injection drug users. J Am Pharm Assoc (Wash). 1999;39(1):23-26.

17. Blumenthal W, Springer KW, Jones S, Stark C. Pharmacy student knowledge, attitudes and beliefs about selling syringes to injection drug users. J Am Pharm Assoc. 2002;42(6 (Suppl 2)):S34-S39.

18. Taussig J, Junge B, Burris S, Jones TS, Sterk CE. Individual and structural influences shaping pharmacists' decisions to sell syringes to injection drug users in Atlanta, Georgia. J Am Pharm Assoc (Wash). 2002;42(6 Suppl 2):S40-S45.

19. Stopka T, Garfein R, Ross A, Truax SR. Increasing syringe access and HIV prevention in California: findings from a survey of local health jurisdiction key personnel. J Urban Health. 2007;84(1):116-125.

20. Epidemiology OoHAa. Key Indicators of Health. Los Angeles: Los Angeles County Department of Public Health; 2007.

21. Lewis BA, Koester SK, Bush TW. Pharmacists' attitudes and concerns regarding syringe sales to injection drug users in Denver, Colorado. J Am Pharm Assoc (Wash). 2002;42(6 Suppl 2):S46-S51.

22. Singer M, Baer HA, Scott G, Horowitz S, Weinstein B. Pharmacy access to syringes among injecting drug users: follow-up findings from Hartford, Connecticut. Public Health Rep. 1998;113(suppl 1):81-89.

23. Fuller CM, Ahern J, Vadnai L, et al. Impact of increased syringe access: preliminary findings on injection drug user syringe source, disposal, and pharmacy sales in Harlem, New York. J Am Pharm Assoc (Wash). 2002;42(6 Suppl 2):S77-S82.

24. Rose V. Expanding access to syringes. Focus. 2007;22(3):1-4.

25. Coffin PO, Ahern J, Dorris S, Stevenson L, Fuller C, Vlahov D. More pharmacists in highrisk neighborhoods of New York City support selling syringes to injection drug users. $J$ Am Pharm Assoc (Wash). 2002;42(6 Suppl 2):S62-S67.

26. Finkelstein R, Tiger R, Greenwald R, Mukherjee R. Pharmacy syringe sale practices during the first year of expanded syringe availability in New York City (2001-2002). J Am Pharm Assoc (Wash). 2002;42(6 Suppl 2):S83-S87. 